\title{
Population Screening for Urine Antigens to Detect Asymptomatic Subarachnoid Neurocysticercosis: A Pilot Study in Northern Peru
}

\author{
Ellen McCleery, ${ }^{1}$ Samantha E. Allen, ${ }^{2}$ Luz Maria Moyano, ${ }^{3}$ Ricardo Gamboa, ${ }^{3}$ Percy Vilchez, ${ }^{3}$ Claudio Muro, ${ }^{3}$ Yesenia Castillo, ${ }^{4}$ \\ Pierre Dorny, ${ }^{5}$ Hector H. Garcia, ${ }^{3,4}$ and Seth E. O'Neal ${ }^{2,3,6 \star}$ for the Cysticercosis Working Group in Peru \\ ${ }^{1}$ University of California Davis, Sacramento, California; ${ }^{2}$ Oregon Health \& Science University, Portland, Oregon; ${ }^{3}$ Centro de Salud Global, \\ Tumbes, Peru; ${ }^{4}$ Universidad Peruana Cayetano Heredia, Lima, Peru; ${ }^{5}$ Institute of Tropical Medicine, Antwerp, Belgium; ${ }^{6}$ Portland State University, \\ Portland, Oregon
}

\begin{abstract}
Subarachnoid neurocysticercosis (SANCC) is a severe and progressive brain infection with Taenia solium. We performed a pilot study of noninvasive screening for SANCC in two endemic villages in northern Peru using a urine antigen screen followed by brain magnetic resonance imaging for participants with elevated levels of antigen. Among the 978 participants screened, we identified eight individuals with SANCC, many of whom were asymptomatic. This represents a minimum prevalence of $0.8 \%$ of SANCC, a level higher than expected based on prior studies, and a positive predictive value of $62 \%$ for our novel urine screening test. Future studies should confirm whether early detection and management improve clinical outcomes.
\end{abstract}

Subarachnoid neurocysticercosis (SANCC) is a progressive and frequently lethal form of central nervous system infection with Taenia solium. Subarachnoid neurocysticercosis occurs when larval cysts implant within the subarachnoid spaces of the basal cisterns, cerebral sulci, Sylvian fissures, and interhemispheric spaces, allowing the parasite to envelope critical structures including the brainstem and intracranial arteries. ${ }^{1-3}$ Unrestrained parasite growth and the accompanying inflammatory response lead to mass effect, intracranial hypertension, and/or hydrocephalus, resulting in substantial morbidity and mortality. ${ }^{3-6}$ Disease onset is slow and insidious; by the time subarachnoid cysts are extensive enough to produce symptoms prompting evaluation, management options are limited. Prolonged antiparasitic and corticosteroid regimens are necessary. Surgical approaches to treat hydrocephalus are challenging and, in some cases, not possible. Even when possible, these procedures are fraught with complications. $^{7-9}$

Early detection and management of SANCC through population screening may improve outcomes if the diagnosis can be made in early stages of disease. Cysticercosis antigen (Ag)-capture assays have been developed for serum, cerebrospinal fluid, and urine. ${ }^{10-12}$ Although Ag levels are highly elevated in SANCC, they are often low or normal in parenchymal disease. ${ }^{12,13}$ This reflects differing pathophysiology and prognoses, as subarachnoid cysts may grow rapidly and unchecked, whereas parenchymal cyst growth is typically restrained. A screening test that quantifies Ag levels could therefore potentially distinguish SANCC from less severe forms of parenchymal neurocysticercosis (NCC), in which the prognosis is often favorable even without treatment. ${ }^{2}$ Our objective was to conduct a pilot study of noninvasive screening for SANCC, in an endemic population, through detection of cysticercosis Ag in urine.

We conducted the study in two villages in northern Peru (Nuevo Progreso and El Tutumo, population 1813) where $T$. solium is endemic. During household visits, residents aged $\geq 12$ years were enrolled, after providing informed *Address correspondence to Seth E. O'Neal, School of Public Health,
Oregon Health \& Science University, Portland State University, 3181
SW Sam Jackson Park Road, Portland, OR 97239. E-mail: oneals@ ohsu.edu consent, in a protocol approved by the Institutional Review Board of Universidad Peruana Cayetano Heredia. We collected information including age, sex, number of household residents, household type, water source, water treatment, electricity source, toilet availability, number of pigs owned, and corralling practices. Each participant received a 1-L plastic container for collection of their first urine sample the following morning. Then, $50 \mathrm{~mL}$ aliquots were frozen at $-20^{\circ} \mathrm{C}$ within 2 hours of urination and analyzed for cysticercosis Ag using ELISA (Ag-ELISA) based on monoclonal antibody set B158/B60. ${ }^{12}$ Results were expressed as a ratio of the optical density (OD) of the sample divided by the mean OD of eight known negative samples plus 3 SDs.

Non-contrast brain magnetic resonance imaging (MRI) was offered to participants with a urine OD ratio of $\geq 3$ to reduce false-positive results and maximize the positive predictive value (PPV) of screening. Magnetic resonance imaging was conducted using a 1.5-tesla machine with T2, FLAIR, and TrueFISP sequences in axial, coronal, and sagittal planes. A study physician collected clinical history including symptoms associated with NCC. Participants with NCC were referred to the regional public hospital for management.

We contacted 1,632 individuals in 296 different households, of which 978 were eligible and agreed to participate (Figure 1). The median age was 31.5 (range 12-89) years and $47.0 \%$ were female. Most participants had urine OD ratio < 1 ( $n=945$; $97.6 \%)$. Of the 33 participants (3.4\%) with an elevated urine OD ratio, 11 were $\geq 1$ and $<2,5$ were $\geq 2$ and $<3$, and 17 were $\geq 3$. No individual or household characteristics measured were associated with urine $O D$ ratio $\geq 3$ on univariate or multivariate analysis (data not show).

Thirteen participants with urine OD ratio $\geq 3$ (mean 13.56; 95\% Cl: 7.98-19.15) completed an MRI and 10 had evidence of NCC (Table 1, Figure 2). Three had SANCC only, two had viable parenchymal NCC only, and five had evidence of both viable parenchymal NCC and SANCC. Therefore, the minimum prevalence of NCC and SANCC in this population was roughly $1 \%$ (10/978) and $0.8 \%(8 / 978)$, respectively, whereas the PPV of urine $\mathrm{Ag}$ at OD ratio $\geq 3$ was approximately $77 \%$ $(10 / 13)$ for any NCC and 62\% (8/13) for SANCC. The mean urine OD ratio among participants without NCC was lower than that of those with NCC (5.12 versus 16.10; $P=0.07)$. 


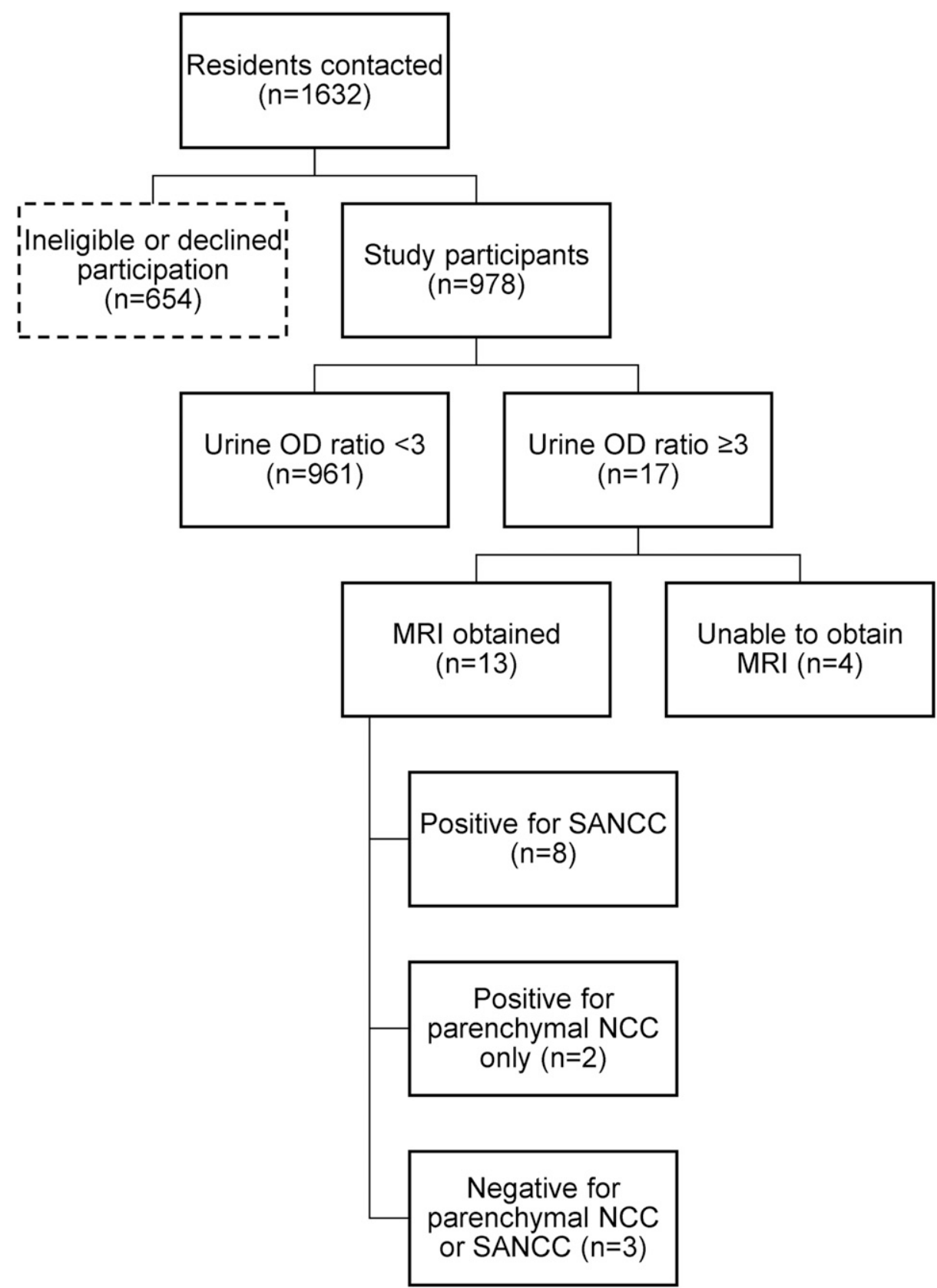

FIGURE 1. Study flowchart showing participation and main study outcomes for pilot study of population screening for cysticercosis antigens in urine to detect SANCC. Optical density ratio: OD ratio for antigen capture using ELISA based on monoclonal antibody set B158/B60. MRI = magnetic resonance imaging (non-contrast) of the brain; NCC = neurocysticercosis; SANCC = subarachnoid neurocysticercosis.

In conclusion, this study demonstrates that population screening for cysticercosis Ag in urine followed by confirmatory brain MRI can identify asymptomatic cases of SANCC. The PPV of urine screening with OD ratio $\geq 3$ was favorable for SANCC $(62 \%)$ and for all forms of NCC $(77 \%)$. This is a novel way to identify individuals with severe forms of NCC, who might otherwise not seek care until they develop symptoms. In addition to its favorable predictive characteristics, this urinebased assay is noninvasive and acceptable to most individuals. Despite these promising initial results, our findings must be replicated on a larger scale, and the optimal cutoff level for urine Ag needs to be determined. In addition, a clinical trial will be necessary to determine whether clinical outcomes of early diagnosis and management are improved through this approach.

We estimated the minimum prevalence of NCC in this population to be at least $1 \%$ and SANCC to be at least $0.8 \%$. This estimate for SANCC, in particular, is higher than we would have expected based on a single study that used MRI to screen for NCC in India, ${ }^{14}$ and prior studies in Peru using computed tomography (CT), ${ }^{15}$ although subarachnoid cysts can be easily missed on CT. The unexpectedly high prevalence of SANCC and lack of associated symptoms highlight our lack of knowledge of the natural history of SANCC in the population setting. It may be that most people with asymptomatic SANCC in the community remain asymptomatic and 
TABLE 1

Clinical characteristics of participants who received non-contrast MRI of the brain, in order from lowest to highest urine OD ratio

\begin{tabular}{|c|c|c|c|c|c|}
\hline Participant & Age (years) & Sex & Symptom & MRI result & Urine OD ratio \\
\hline 1 & 16 & Female & Headache & Negative & 3.60 \\
\hline 2 & 31 & Male & None & Subarachnoid NCC & 4.62 \\
\hline 3 & 21 & Female & None & Negative & 5.81 \\
\hline 4 & 56 & Female & Migraine & Negative & 5.95 \\
\hline 5 & 25 & Male & None & Parenchymal NCC & 6.14 \\
\hline 6 & 37 & Male & History of traumatic brain injury & Subarachnoid NCC & 7.59 \\
\hline 7 & 32 & Male & None & Subarachnoid and parenchymal NCC & 8.68 \\
\hline 8 & 71 & Male & None & Subarachnoid NCC & 15.88 \\
\hline 9 & 66 & Male & None & Subarachnoid and parenchymal NCC & 16.56 \\
\hline 10 & 64 & Male & History of traumatic brain injury & Subarachnoid and parenchymal NCC & 20.65 \\
\hline 11 & 67 & Male & Headache & Parenchymal NCC & 24.27 \\
\hline 12 & 39 & Male & Epilepsy & Subarachnoid and parenchymal NCC & 27.57 \\
\hline 13 & 33 & Male & Headache & Subarachnoid and parenchymal NCC & 28.99 \\
\hline
\end{tabular}

that progressive disease seen in clinical settings is a biased sample. Conversely, given the lack of access to health care and neuroimaging in most rural endemic settings, it is also possible that people with SANCC may succumb to their disease without receiving a diagnosis. Understanding natural progression is important to determine whether a screening approach is warranted.

Three participants with high urine Ag levels did not have NCC on MRI. This might be due to cysts infecting areas of the body that we did not image, missed brain cysts on MRI, or false-positive cases in which $T$. solium infection is not present. It is possible that cross-reactivity with other parasitic
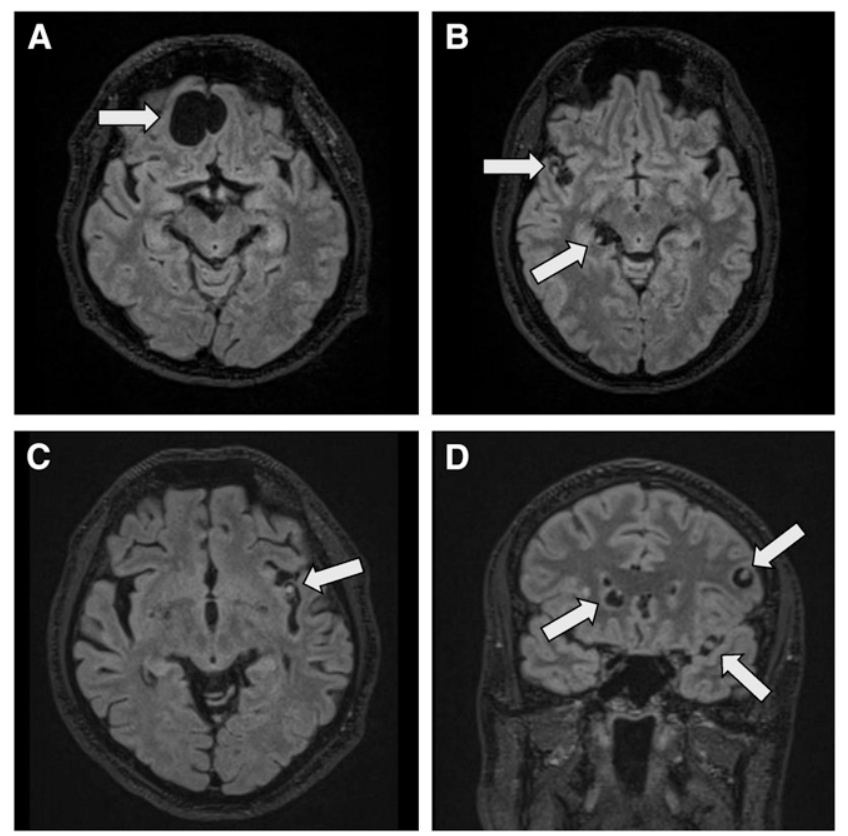

FIGURE 2. FLAIR sequence images obtained using non-contrast magnetic resonance imaging, showing representative subarachnoid and parenchymal neurocysticercosis from four participants with high levels of cysticercosis antigen (Ag) in the urine on ELISA (B158/B60). (A) Axial image showing a multilocular interhemispheric subarachnoid cyst (optical density [OD] ratio 16.56), (B) axial image showing perimesencephalic and Sylvian fissure subarachnoid cysts (OD ratio 15.88), (C) axial image showing a single Sylvian fissure subarachnoid cyst (OD ratio 8.68), and (D) coronal image showing a Sylvian fissure subarachnoid cyst along with multiple parenchymal cysts (OD ratio 27.57). infections, or potentially with host-excreted proteins, could be responsible for false-positive results. In addition, the variability of urine $\mathrm{Ag}$ levels over time has not been characterized among people with NCC, and this could influence screening results. Future longitudinal studies are warranted to evaluate these factors.

This study has limitations. First, as this is a small pilot study in two Peruvian villages, results may not generalize to other settings. Second, we limited MRI to participants with high urine Ag levels; we cannot be sure that we identified all participants with NCC, nor can we comment on assay sensitivity and specificity. Larger studies are needed to confirm our findings, as well as longitudinal studies that characterize variability in urine Ag excretion, natural history of SANCC, and effectiveness of early detection and management. Our research group has studies underway that address these limitations and knowledge gaps. Ultimately, we aim to determine the effectiveness and utility of a population screening approach to improve clinical prognosis of SANCC.

Received March 16, 2020. Accepted for publication May 20, 2020.

Published online June 29, 2020.

Acknowledgments: We are grateful for the operational support of the research staff at the Center for Global Health Tumbes and for the participation of residents from Nuevo Progreso and El Tutumo.

Financial support: This research was made possible with support from the National Institute of Neurological Disorders and Stroke (NINDS) and the Fogarty International Center (FIC) under the Brain Disorders in the Developing World: Research Across the Lifespan program (Grant R01NS103623), and the Fogarty Global Infectious Disease Research Training Program (D43TW001140).

Disclosure: This study was reviewed and approved by the institutional review boards at Universidad Peruana Cayetano Heredia and at Oregon Health \& Sciences University

Disclaimer: The opinions expressed by authors contributing to this article do not necessarily reflect the opinions of the Centers for Disease Control and Prevention, the institutions with which the authors are affiliated, or the funding agency. The design and interpretation of this study were done solely by the authors.

Authors' addresses: Ellen McCleery, University of California Davis, Sacramento, CA, E-mail: ellen.mccleery@gmail.com. Samantha E. Allen, Oregon Health \& Science University, Portland, OR, E-mail: allensam@ohsu.edu. Luz Maria Moyano, Ricardo Gamboa, Percy Vilchez, and Claudio Muro, Centro de Salud Global, Tumbes, Peru, E-mails: luzmariamoyano@gmail.com, rgamboa@peruresearch.org, pvilchez@peruresearch.org, and claudio.muro@peruresearch.org. Yesenia Castillo, Universidad Peruana Cayetano Heredia, Lima, Peru, E-mail: elisa_ip@peruresearch.org. Pierre Dorny, Institute of Tropical 
Medicine, Antwerp, Belgium, E-mail: pdorny@itg.be. HectorH. Garcia, Centro de Salud Global, Tumbes, Peru and Universidad Peruana Cayetano Heredia, Lima, Peru, E-mail: hgarcia1@jhu.edu. Seth E. O'Neal, Oregon Health \& Science University, Portland, OR and Portland State University, Portland, OR, E-mail: oneals@ohsu.edu.

\section{REFERENCES}

1. Proaño JV, Madrazo I, Avelar F, López-Félix B, Díaz G, Grijalva I, 2001. Medical treatment for neurocysticercosis characterized by giant subarachnoid cysts. N Engl J Med 345: 879-885.

2. Estañol B, Corona T, Abad P, 1986. A prognostic classification of cerebral cysticercosis: therapeutic implications. I Neurol Neurosurg Psychiatry 49: 1131-1134.

3. Fleury A, Carrillo-Mezo R, Flisser A, Sciutto E, Corona T, 2011. Subarachnoid basal neurocysticercosis: a focus on the most severe form of the disease. Expert Rev Anti Infect Ther 9: 123-133.

4. Nash TE, O'Connell EM, Hammoud DA, Wetzler L, Ware JM, Mahanty S, 2020. Natural history of treated subarachnoid neurocysticercosis. Am J Trop Med Hyg 102: 78-89.

5. Sotelo J, Marin C, 1987. Hydrocephalus secondary to cysticercotic arachnoiditis: a long-term follow-up review of 92 cases, 1987. J Neurosurg 66: 686-689.

6. Cuetter AC, Andrews RJ, 2002. Intraventricular neurocysticercosis: 18 consecutive patients and review of the literature. Neurosurg Focus 12: 1-7.

7. Proaño JV, Torres-Corzo J, Rodríguez-Della Vecchia R, GuizarSahagun G, Rangel-Castilla L, 2009. Intraventricular and subarachnoid basal cisterns neurocysticercosis: a comparative study between traditional treatment versus neuroendoscopic surgery. Childs Nerv Syst 25: 1467-1475.

8. Kelley R, Duong DH, Locke GE, 2002. Characteristics of ventricular shunt malfunctions among patients with neurocysticercosis. Neurosurgery 50: 757-762.

9. Nash TE, Mahanty S, Garcia HH, 2011. Corticosteroid use in neurocysticercosis. Expert Rev Neurother 11: 1175-1183.

10. Brandt JRA, Falla N, Ceulemans F, Brijs L, 1992. Antibody-based elisa for the detection of excretory-secretory antigens in Taenia saginata cysticercosis. Int J Parasitol 22: 471-477.

11. Garcia HH et al., 1998. A specific antigen-detection elisa for the diagnosis of human neurocysticercosis. Trans $R$ Soc Trop Med Hyg 92: 411-414.

12. Castillo $Y$ et al., 2009. Urine antigen detection for the diagnosis of human neurocysticercosis. Am J Trop Med Hyg 80: 379-383.

13. Rodriguez $S$ et al., 2009. Detection of Taenia solium antigens and anti- $T$. solium antibodies in paired serum and cerebrospinal fluid samples from patients with intraparenchymal or extraparenchymal neurocysticercosis. J Infect Dis 199: 1345-1352.

14. Prasad KN, Verma A, Srivastava S, Gupta RK, Pandey CM, Paliwal VK, 2011. An epidemiological study of asymptomatic neurocysticercosis in a pig farming community in northern India. Trans R Soc Trop Med Hyg 105: 531-536.

15. Moyano LM et al., 2016. High prevalence of asymptomatic neurocysticercosis in an endemic rural community in Peru. PLoS Negl Trop Dis 10: e0005130. 